\title{
Robust conductance of dumbbell molecular junctions with fullerene anchoring groups
}

\author{
Markussen, Troels; Settnes, Mikkel; Thygesen, Kristian Sommer
}

Published in:

Journal of Chemical Physics

Link to article, DOI:

$10.1063 / 1.3646510$

Publication date:

2011

Document Version

Publisher's PDF, also known as Version of record

Link back to DTU Orbit

Citation (APA):

Markussen, T., Settnes, M., \& Thygesen, K. S. (2011). Robust conductance of dumbbell molecular junctions with fullerene anchoring groups. Journal of Chemical Physics, 135(14), 144104. https://doi.org/10.1063/1.3646510

\section{General rights}

Copyright and moral rights for the publications made accessible in the public portal are retained by the authors and/or other copyright owners and it is a condition of accessing publications that users recognise and abide by the legal requirements associated with these rights.

- Users may download and print one copy of any publication from the public portal for the purpose of private study or research.

- You may not further distribute the material or use it for any profit-making activity or commercial gain

- You may freely distribute the URL identifying the publication in the public portal 


\section{AIP chemicalit Physics}

\section{Robust conductance of dumbbell molecular junctions with fullerene anchoring groups}

Troels Markussen, Mikkel Settnes, and Kristian S. Thygesen

Citation: J. Chem. Phys. 135, 144104 (2011); doi: 10.1063/1.3646510

View online: http://dx.doi.org/10.1063/1.3646510

View Table of Contents: http://jcp.aip.org/resource/1/JCPSA6/v135/i14

Published by the American Institute of Physics.

\section{Related Articles}

X-ray photoelectron spectroscopy investigation of the Schottky barrier at low-k a-SiO(C):H/Cu interfaces Appl. Phys. Lett. 99, 202903 (2011)

Influence of $\mathrm{Al} / \mathrm{CuO}$ reactive multilayer films additives on exploding foil initiator

J. Appl. Phys. 110, 094505 (2011)

A comparative study of the electrical properties of $\mathrm{Pd} / \mathrm{ZnO}$ Schottky contacts fabricated using electron beam deposition and resistive/thermal evaporation techniques

J. Appl. Phys. 110, 094504 (2011)

Charge transfer dynamics of 3,4,9,10-perylene-tetracarboxylic-dianhydride molecules on $\mathrm{Au}(111)$ probed by resonant photoemission spectroscopy

J. Chem. Phys. 135, 174701 (2011)

Schottky barrier height tuning of silicides on p-type Si (100) by aluminum implantation and pulsed excimer laser anneal

J. Appl. Phys. 110, 073703 (2011)

\section{Additional information on J. Chem. Phys.}

Journal Homepage: http://jcp.aip.org/

Journal Information: http://jcp.aip.org/about/about_the_journal

Top downloads: http://jcp.aip.org/features/most_downloaded

Information for Authors: http://jcp.aip.org/authors

\section{ADVERTISEMENT}

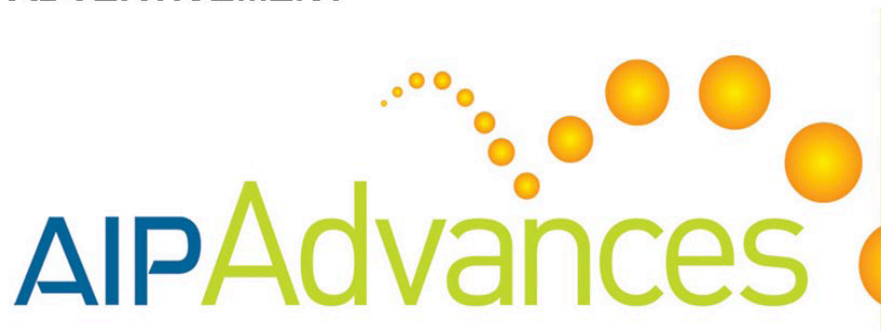

Explore AIP's new

open-access journal

Article-level metrics now available

Join the conversation!

Submit Now
Rate \& comment on articles 


\title{
Robust conductance of dumbbell molecular junctions with fullerene anchoring groups
}

\author{
Troels Markussen, ${ }^{\text {a) }}$ Mikkel Settnes, and Kristian S. Thygesen \\ Center for Atomic-scale Materials Design (CAMD), Department of Physics, Technical University of Denmark, \\ DK-2800 Kgs. Lyngby, Denmark
}

(Received 29 April 2011; accepted 15 September 2011; published online 11 October 2011)

\begin{abstract}
The conductance of a molecular wire connected to metallic electrodes is known to be sensitive to the atomic structure of the molecule-metal contact. This contact is to a large extent determined by the anchoring group linking the molecular wire to the metal. It has been found experimentally that a dumbbell construction with $\mathrm{C}_{60}$ molecules acting as anchors yields more well-defined conductances as compared to the widely used thiol anchoring groups. Here, we use density functional theory to investigate the electronic properties of this dumbbell construction. The conductance is found to be stable against variations in the detailed bonding geometry and in good agreement with the experimental value of $\mathrm{G}=3 \times 10^{-4} \mathrm{G}_{0}$. Electron tunneling across the molecular bridge occurs via the lowest unoccupied orbitals of $\mathrm{C}_{60}$ which are pinned close to the Fermi energy due to partial charge transfer. Our findings support the original motivation to achieve conductance values more stable towards changes in the structure of the molecule-metal contact leading to larger reproducibility in experiments. (c) 2011 American Institute of Physics. [doi:10.1063/1.3646510]
\end{abstract}

\section{INTRODUCTION}

Electron transport junctions consisting of single molecules sandwiched between metallic electrodes have received significant theoretical and experimental attention over the past decade. ${ }^{1-4}$ Such junctions are the smallest possible electrical conductors and represent the most elementary device within molecular electronics. One of the main problems in this field is that experiments often suffer from large fluctuations in the measured conductance originating from variations in the atomic details in the bonding geometry beyond experimental control. The challenge is to create a molecular wire with anchoring groups giving a well-defined and robust interface to the metallic electrodes. ${ }^{5}$ The most common approach is to use thiol anchoring groups which adhere strongly to a gold surface. However, the large strength of the Au-S bond can lead to many different binding configurations, which again is reflected in the electronic properties of the molecular junction. ${ }^{6-13}$

To overcome this problem, it has recently been proposed to use $\mathrm{C}_{60}$ molecules as anchors ${ }^{14,15}$ to obtain a more welldefined bonding to the gold electrodes. It is well known that $\mathrm{C}_{60}$ hybridizes strongly with gold surfaces ${ }^{16}$ providing a good electrical contact. At the same time the size and symmetry of the $\mathrm{C}_{60}$ molecules should reduce the sensitiveness to the details of the contact geometry. Thus, the hope is that the $\mathrm{C}_{60}$ anchors would effectively function as an integrated part of the electrodes giving a well-defined reproducible charge injection into a molecular core. It was indeed experimentally demonstrated that 1,4-bis(fullero[c]pyrrolidin1-yl)benzene (BDC60) with $\mathrm{C}_{60}$ anchor groups yields more stable conductances than similar thiol-bonded molecules. ${ }^{14}$ Elementary theoretical calculations for the BDC60 junction

\footnotetext{
a)Electronic mail: trma@ fysik.dtu.dk.
}

were presented in Ref. 15. However, the question of contact robustness was not addressed nor was the calculated conductance values reported.

In this paper, we focus on the dumbbell construction BDC60 between Au(111) electrodes, shown in Fig. 1. Using density functional theory (DFT) in combination with nonequilibrium Green functions, we show that the conductance of BDC60 is relatively stable with respect to the details of the bonding geometry. Furthermore, the calculated conductance is in good agreement with the experimental value of $G$ $=3 \times 10^{-4} \mathrm{G}_{0} \cdot{ }^{14}$ The quantitative agreement between DFT and experiments is shown to be a consequence of the lowest unoccupied molecular energy levels being strongly hybridized with the gold electrodes and pinned close to the Fermi level by charge transfer.

\section{METHODS}

We study the transport properties of molecular junctions with a BDC60 molecule, shown in Figs. 1(a) and 1(b). The structure of the free molecule has been relaxed using the GPAW electronic structure package which is a real space implementation of the projector-augmented wave method. ${ }^{17}$

In the molecular junction, the BDC60 is connected to two $\mathrm{Au}(111)$ surfaces. We consider a total number of 30 junction geometries in order to address the impact on the conductance. The relaxed BDC60 is placed on three different sites indicated by Figs. 1(c) and 1(e). The names of the sites (hcp, bridge, and top) correspond to the position of the center of the end-hexagon. In addition to the hexagon orientation shown in Figs. 1(c) and 1(e), we also consider the so-called 6:6 orientation where two carbon atoms forming the bond between two hexagons face the gold surface. ${ }^{18}$ For each orientation and for each binding site we rotate the molecule in five steps 
(a)

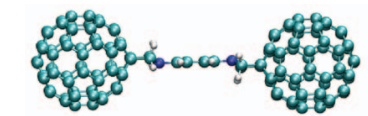

(c)

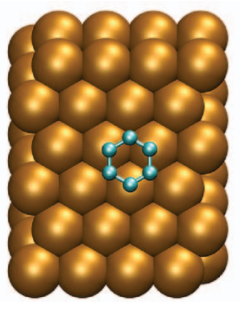

(d)

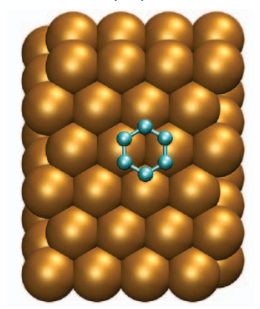

(f)

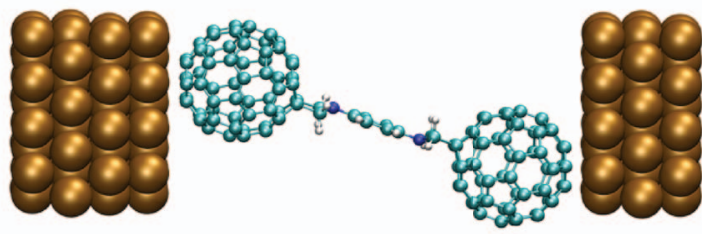

(g)
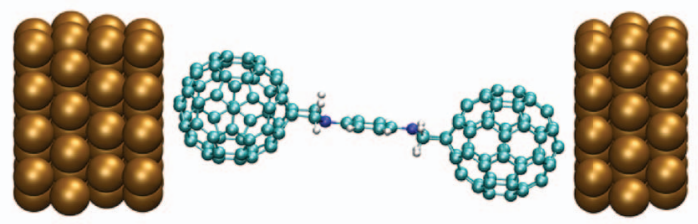

FIG. 1. Structure of the BDC60 molecule ((a) and (b)). Panels (c)-(e) show the position of the end-hexagon of the BDC60 on the Au (111) surface: (c) hexagonal center position (hcp), (d) bridge position, (e) top position (the names refer to the center of the hexagon). The $(3 \times 4) \mathrm{Au}(111)$ surface corresponds to the transverse size of the supercell in the DFT and transport calculations. The transport supercell is shown in panel (f) for a hexagon adsorbed molecule and in panel $(\mathrm{g})$ for a $6: 6$ bond adsorbed molecule.

between $0^{\circ}$ and $60^{\circ}$. The bonding distance between $\mathrm{C}_{60}$ and $\mathrm{Au}$ was found to be $2.4 \AA$ in Ref. 19 using the local density approximation (LDA), and we adopt this value for the distance between the BDC60 molecule and the flat $\mathrm{Au}(111)$. The distance refers to the distance between the $\mathrm{Au}(111)$ surface and the closest $\mathrm{C}$ atom. We note that both experiments ${ }^{20}$ and calculations ${ }^{19}$ show that the $\mathrm{Au}-\mathrm{C}_{60}$ bond is covalent with some ionic character and with a measured charge transfer of 0.8 electrons per $\mathrm{C}_{60} .{ }^{20}$ Dispersive van-der-Waals interactions between $\mathrm{C}_{60}$ and $\mathrm{Au}$ are thus not relevant and the binding should be well described with standard DFT in the LDA, as used in Ref. 19.

For both the hexagon and $6: 6$ orientations, the left $\mathrm{C}_{60}$ molecule is placed symmetrically on either the hcp, bridge, or top site, while the right $\mathrm{C}_{60}$ may be placed slightly asymmetrically. In all calculations we use a $(3 \times 4)$ Au cell as shown in Fig. 1 with a total of 7 Au layers.

The conductance through the scattering region is calculated within the Landauer-Büttiker formalism as $\mathrm{G}=\mathrm{G}_{0} T\left(E_{F}\right)$, where $\mathrm{G}_{0}=2 e^{2} / h$ is the fundamental conductance quantum and $T\left(E_{F}\right)$ is the transmission function evaluated at the Fermi level. ${ }^{21}$ The zero-bias transmission function is calculated using a standard Green function method
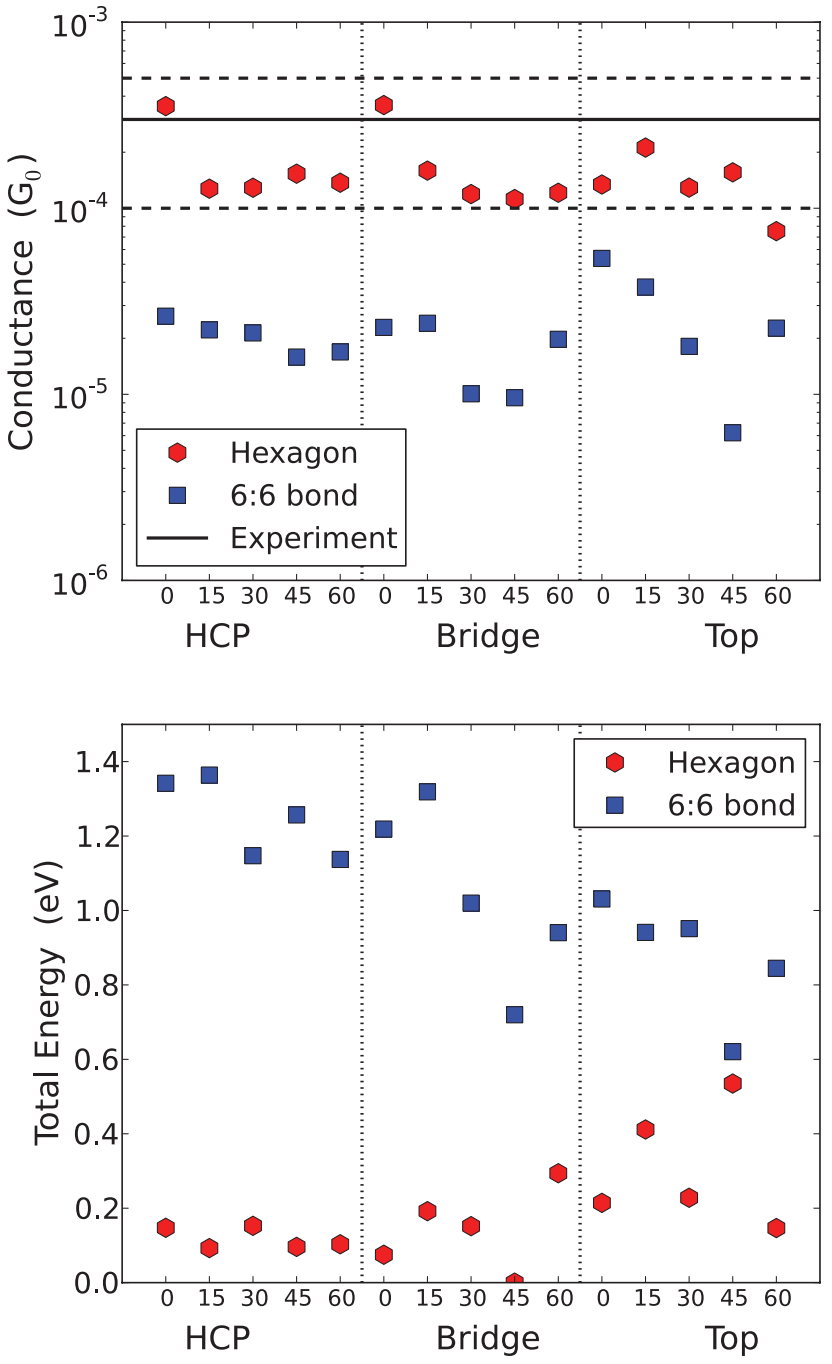

FIG. 2. Top: Calculated conductance values (transmission at the Fermi level) for all the considered geometries. The numbers on the abscissa denote the rotation angles. The structures with hexagon binding (red hexagons) are all within the experimental uncertainty (horizontal dashed lines), whereas the structures adsorbed on 6:6 bonds give systematically lower conductances. Bottom: Total energies (relative to the minimum energy) for all (unrelaxed) geometries.

as described in Ref. 22. The Kohn-Sham Hamiltonian of the scattering region is obtained using a double- $\zeta$ polarized basis $\mathrm{set}^{23}$ for all the atoms. The calculations are performed with a $(4,4,1)$ k-point sampling and the exchange correlation potential described by the Perdew-Burke-Ernzerhof functional. ${ }^{24}$

\section{RESULTS}

Figure 2 (top) shows the conductance for all the considered geometries. The average experimental value of $3 \times 10^{-4} \mathrm{G}_{0}$ is indicated by the solid line, while the dashed lines mark estimated uncertainties in the experimental values. ${ }^{14}$ We observe that the conductance values for the hexagon adsorbed molecules lie relatively close to the experimental value (within a factor of three) while adsorption on the 6:6 bond gives roughly an order of magnitude lower conductance. We also notice that within each type of adsorption geometry, the conductance remains fairly constant when 


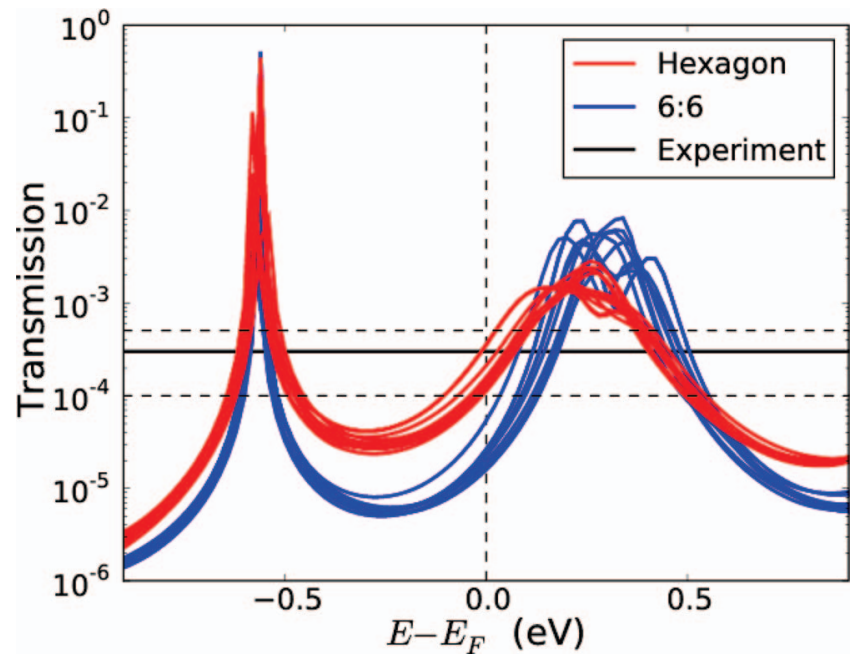

FIG. 3. Transmission functions on a log-scale vs. energy for hexagon adsorbed molecules (red) and 6:6 bond adsorption (blue).

the binding site and rotation angle is varied. This shows that the fullerene anchor groups indeed are quite insensitive to the details in the contact geometry, as experimentally indicated. ${ }^{14}$

In Fig. 2 (bottom), we show the calculated total energies for all the geometries. We find that the hexagon adsorbed molecules on average have $\sim 1 \mathrm{eV}$ lower energies than those adsorbed on the 6:6 bond. Although the energies are calculated for unrelaxed geometries, this indicates that the hexagon adsorption geometry would be energetically the most favorable - at least at the considered bonding distance of $2.4 \AA$.

The transmission functions for the different sites and rotations are shown in Fig. 3. It is evident that not only the conductance (transmission at the Fermi level) is robust against junction geometry, but the entire energy-dependent transmission function varies only little among the different sites and rotations (within each adsorption type). This results further support the hypothesis that the fullerenes are good candidates for robust anchor groups.

From the transmission functions in Fig. 3 we see that the origin of the lower conductance values of the 6:6 adsorbed molecules is the more narrow transmission peaks. Both the high transmission peak below the Fermi energy and the broad peak right above the Fermi energy are significantly more narrow for the 6:6 adsorbed molecules. The smaller broadening for the 6:6-bond geometries may simply be explained by a weaker coupling to the Au electrodes: While the 6:6bond mainly couples to the $\mathrm{Au}(111)$ surface via two carbon atoms per $\mathrm{C}_{60}$, the hexagon adsorbed molecules couple via 6 carbon atoms per $\mathrm{C}_{60}$. The higher coordination number for the hexagon adsorption geometry enhances the effective electronic coupling leading to more broadened transmission peaks and higher conductance. While the energy of the hexagon bonding configuration is minimal around $2.4 \AA$, the optimal 6:6 configuration may be closer to the $\mathrm{Au}(111)$ surface due to its weaker coupling. This would in turn increase the conductance values of the 6:6 configuration bringing them in closer agreement with those of the hexagon and experimental values (see Fig. 2 (top)).

\section{A. Molecular orbital analysis}

For all the considered geometries, the Fermi level, $E_{F}$, is located between two peaks of different character: A narrow transmission peak centered at $E-E_{F}=-0.6 \mathrm{eV}$ with peak values of 0.1-0.5 and a much broader transmission peak centered around $E-E_{F}=0.25 \mathrm{eV}$ with significantly lower peak values of $0.001-0.01$. To further analyze the origin of the two transmission peaks, we proceed by calculating the eigenstates of the BDC60 molecule in the junction. From the full Hamiltonian, $\mathbf{H}$, and overlap matrix, $\mathbf{S}$, of the combined Au-molecule-Au system, we project onto the subspace spanned by the basis functions of the molecule (BDC60):

$$
\mathbf{h}_{\mathrm{mol}}=\mathbf{P}_{\mathrm{mol}} \mathbf{H} \mathbf{P}_{\mathrm{mol}} \text {. }
$$

Here $\mathbf{P}_{\text {mol }}$ is a projection matrix with unit diagonal elements on the indices of the molecule basis functions, and zeros elsewhere. Similarly we get a molecule overlap matrix, $\mathbf{s}_{\mathrm{mol}}$. We then solve the (generalized) eigenvalue problem

$$
\mathbf{h}_{\mathrm{mol}} \mathbf{c}_{\mathrm{n}}=\varepsilon_{n} \mathbf{s}_{\mathrm{mol}} \mathbf{c}_{\mathrm{n}}
$$

to find the energies $\left(\varepsilon_{n}\right)$ and eigenstates $\left(\mathbf{c}_{\mathrm{n}}\right)$ of the molecule. We emphasize that in this procedure, the eigenenergies are calculated with respect to the Au Fermi energy and include charge transfer effects between the BDC60 and the Au electrodes. This information would not be available in the calculation of the free molecule without the Au electrodes.

Figure 4 shows the frontier orbitals relevant for the transmission around the Fermi energy. It is evident that the narrow transmission peak around $E-E_{F}=-0.6 \mathrm{eV}$ is associated with the HOMO eigenstate located on the central part of the BDC60. Since this state has vanishing orbital weight close to the $\mathrm{Au}$ electrodes, the broadening due to the electrodes
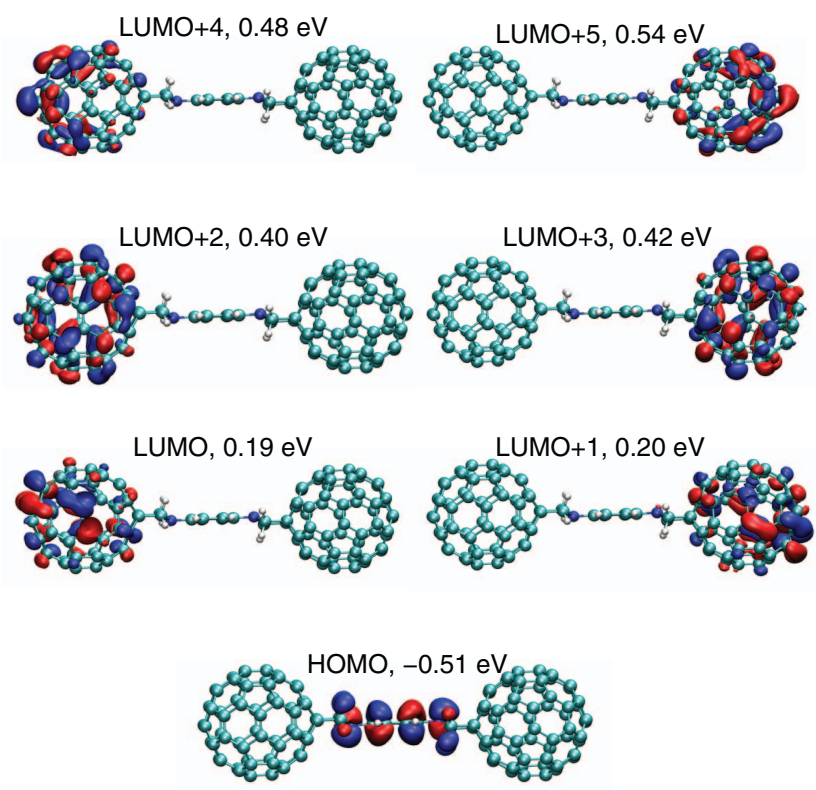

FIG. 4. Eigenstates of the molecule in the junction. These are obtained from the full transport setup including the Au electrodes by first projecting onto the molecular basis orbitals and diagonalizing the resulting Hamiltonian. The HOMO is responsible for the narrow transmission peaks around $E-E_{F}$ $=-0.6 \mathrm{eV}$ in Fig. 3, while the six states LUMO to LUMO +5 are responsible for the broad transmission peaks around $E-E_{F}=0.3 \mathrm{eV}$. 


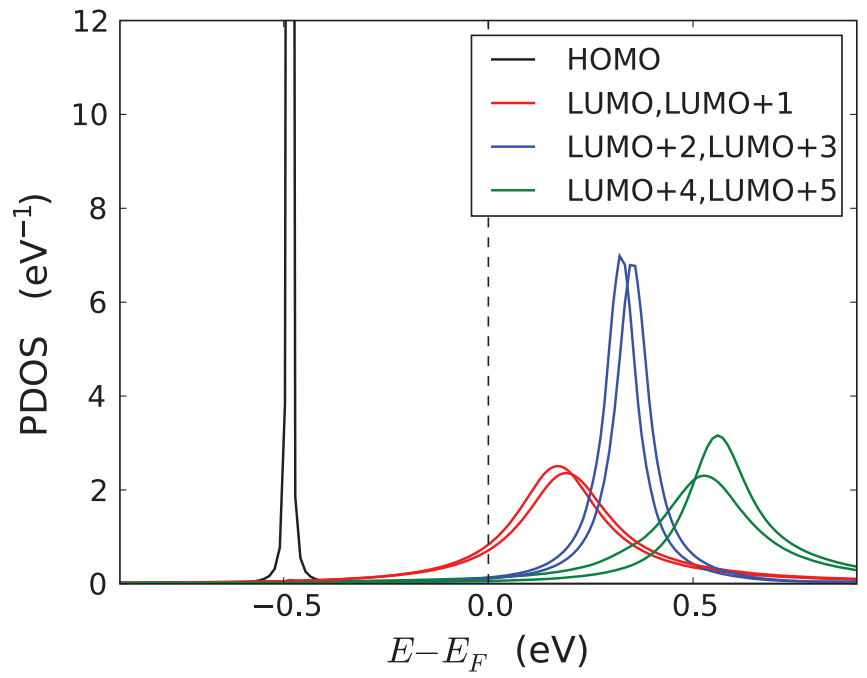

FIG. 5. Projected density of states on the HOMO (solid black) and on the LUMO-LUMO + 5 eigenstates of the molecule in the junction (cf. Fig. 4). The HOMO state located on the central part is seen as a very localized peak around $E-E_{F}=-0.5 \mathrm{eV}$. The six states LUMO to LUMO +5 are significantly broader having finite weights at the Fermi energy. The contribution to the total electron density from the LUMO-LUMO + 5 amounts to 0.5 electron. This indicates that the LUMO peak is pinned close to the Fermi level.

is very weak, resulting in a narrow transmission peak. To further stress that the narrow transmission peak originates from the HOMO state, we show in Fig. 5 the projected density of states (PDOS) on the HOMO orbital (solid black). Again, we observe a narrow peak around $E-E_{F}=-0.5 \mathrm{eV}$ with a clear correspondence to the transmission function.

The broad transmission peak around $E-E_{F}=0.3 \mathrm{eV}$ is associated with the six eigenstates, LUMO to LUMO +5 . These states come in nearly degenerate symmetric pairs and are mainly located on the $\mathrm{C}_{60}$ part of the BDC60 molecule. We note that isolated $\mathrm{C}_{60}$ has a three-fold degenerate LUMO ${ }^{25}$ and the six lowest unoccupied states in the BDC60 are hence derived from the six LUMOs from the two $\mathrm{C}_{60}$ molecules (three from each). The strict localization in the left/right part of each pair signals a weak electronic coupling between the two $\mathrm{C}_{60}$. Each of these states has a large orbital weight close to the $\mathrm{Au}$, and the broadening due to the electrodes is thus expected to be large, resulting in a broad transmission peak, as indeed observed in Fig. 3. Since the states are mainly located on one of the $\mathrm{C}_{60}$ parts, they do not contribute with a large transmission, since the electrons that enter the BDC60 in one end (in a state with large orbital weight and strong coupling) cannot leave it again in the other end due the low orbital weight and corresponding weak coupling. This is the reason why the broad transmission peak has a low peak value.

The analysis of molecular orbitals explains the relatively low conductance of $3 \times 10^{-4} \mathrm{G}_{0}$. The symmetrically placed and highly transmitting HOMO state is only weakly coupled to the Au electrodes resulting in a very narrow transmission peak with a small overlap with the Fermi energy. The six lowest unoccupied states are strongly, but highly asymmetrically, coupled to the electrodes leading to broad transmission peaks with low peak values.

\section{DISCUSSION}

In the remainder of the paper, we discuss the, perhaps surprisingly, good agreement between the measured conductance and our calculations, and analyze the robustness of our results. It is well known ${ }^{26-31}$ that DFT often fails to properly describe molecular level positions. This often leads to calculated conductance values which are orders of magnitudes larger than the experimental results. A number of theoretical works have proposed alternative ways of calculating the conductance beyond DFT. ${ }^{26-30,32,33}$

Often the large discrepancies between experiments and DFT can be explained by a too small calculated ionization potential (IP) (for HOMO dominated transport) or too large electron affinity (for LUMO dominated transport). ${ }^{32,33}$ If the HOMO and LUMO levels are far away from the Fermi energy (compared to the level broadening), there is no reason to believe that DFT will give the correct level position, since the total electron density does not change, if the levels are shifted up or down in energy. This is indeed the case for the HOMO state in our calculations. From the PDOS plot in Fig. 5 it is evident that the contribution to the electron density (integrated PDOS up to the Fermi energy) from the HOMO (solid black) does not change, if the peak around $E-E_{F}=-0.5 \mathrm{eV}$ is shifted down in energy.

On the other hand, the PDOS peaks from particularly the LUMO and LUMO + 1 (red) have significant weights below the Fermi energy. The contribution to the total electron density from the LUMO to LUMO +5 is around 1 electron assuming spin degeneracy. Since the total electron density in principle is correctly described with DFT, an upward shift of the unoccupied states would change the (in principle) correct density, leading to a wrong result. The lowest unoccupied states are, thus, pinned close to the Fermi level and the energy levels can be expected to be reasonably well described at the DFT level of theory. We note that the calculated charge transfer of $\sim 1.0$ electrons $\left(0.5\right.$ electrons per $\left.\mathrm{C}_{60}\right)$ is in qualitative agreement with previous calculations on $\mathrm{C}_{60}$ molecules where a charge transfer of 0.2 electrons per $\mathrm{C}_{60}$ molecule was reported. ${ }^{19}$ We further note that when we calculate the charge transfer for a very asymmetric junction with a large vacuum gap between one of the $\mathrm{C}_{60}$ and the neighboring $\mathrm{Au}$ surface, we obtain a charge transfer of 0.52 electrons. This shows that the charge transfer to one $\mathrm{C}_{60}$ is largely independent of the electrode coupling to the other $\mathrm{C}_{60}$. Finally, the calculated charge transfer is in agreement with experiments showing a charge transfer of $\sim 0.8$ electrons per $\mathrm{C}_{60}$ on $\mathrm{Au},{ }^{20}$ thus further indicating that the DFT calculated LUMO position is accurate. Since the LUMO (and LUMO + 1) are mainly responsible for the conductance, it follows that the DFT calculated conductance also is expected to be reasonably accurate. The close agreement between our calculated conductance values and the experimental results further supports this.

As mentioned above, the position of the HOMO level might be incorrectly calculated with DFT being too close to the Fermi level. In order to address the effect of the HOMO position, we have carried out DFT $+\Sigma_{\text {occ }}$ calculations, ${ }^{32,34}$ where an extra self-energy term, $\Sigma_{\text {occ }}$, acts on the occupied states on the molecule by shifting them down in energy. The 


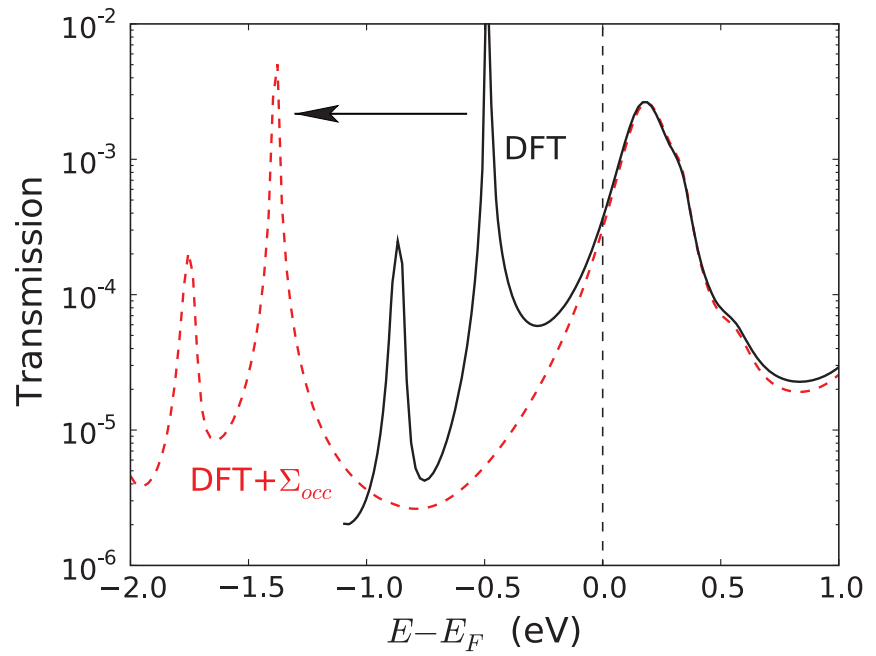

FIG. 6. Transmission function calculated with DFT (solid) and with DFT $+\Sigma_{\text {occ }}$ (dashed). The transmission at the Fermi energy is only slightly reduced when the occupied states are shifted down in energy.

energy shift $\Sigma_{\text {occ }}=-0.9 \mathrm{eV}$ is composed of two terms: (i) A correction $\Delta_{0}=-1.4 \mathrm{eV}$ to shift the Kohn-Sham HOMO level of the free molecule down in energy to match the IP obtained from total energy differences between the neutral and positively charged (+1e) molecule. (ii) An upward shift $\Delta_{q} \simeq 0.5 \mathrm{eV}$ due to image charge corrections. ${ }^{34}$ Figure 6 compares the transmission functions obtained with DFT (as all the results above) and with DFT $+\Sigma_{\text {occ }}$. We observe that even if the HOMO state is moved down, the conductance (transmission at $E=E_{F}$ ) only changes marginally, and we thus conclude that the conductance is completely governed by transport through the unoccupied LUMO to LUMO +5 states.

We note that in recent single-molecule experiments on dumbbell molecules with $\mathrm{C}_{60}$ anchor groups, ${ }^{35}$ very similar to the BDC60 studied here, the conductance was found to be on the order of $10^{-4} \mathrm{G}_{0}$. This value is quite close to that measured for BDC60 and predicted for BDC60 by our calculations. Based on the similarity of conductance values and on preliminary calculations, we expect the physics of the dumbbell molecule junctions investigated in Ref. 35 to be very similar to the one studied here. In particular, we expect the conductance to be governed by low-transmitting, asymmetric LUMO orbitals of the $\mathrm{C}_{60}$.

The distance between the BDC60 and the Au surface is a source of uncertainty. In the above calculations, we have used the equilibrium distance of $2.4 \AA$ as obtained for $\mathrm{C}_{60} \cdot{ }^{19}$ To address the dependence on bonding distance, we show in Fig. 7 the transmission function of the BDC60 molecules for $\mathrm{C}_{60}$-Au distances of $2.2 \AA$ (solid black), $2.4 \AA$ (dashed-dotted red), and $2.6 \AA$ (dashed blue). It is seen that the transmission function at the Fermi energy is increased by a factor of two when the bonding distance is reduced to $2.2 \AA$, while it is reduced by a factor of 7 , when the distance is increased to 2.6 ̊. An increase in $\mathrm{C}_{60}$ - $\mathrm{Au}$ distance leads to more narrow transmission peaks due to weaker couplings. In addition, the HOMO level moves down in energy, while the unoccupied levels move up in energy. Both these effects contribute to the

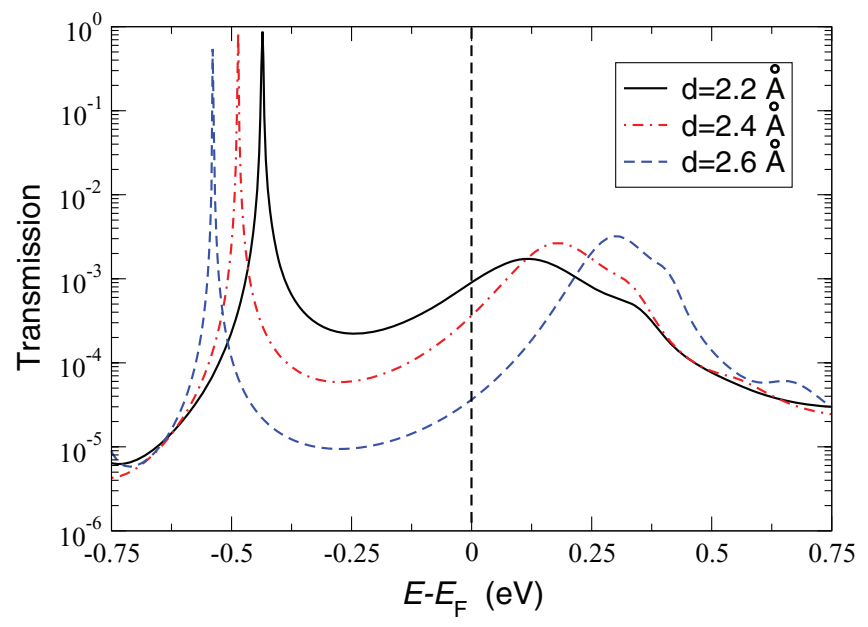

FIG. 7. Transmission function at three different distances between the endhexagons on the $\mathrm{C}_{60}$ and the Au surface.

lower conductance values. Within the bond length variations between 2.2 and $2.6 \AA$, the conductance is thus changed between $8 \times 10^{-4} \mathrm{G}_{0}$ and $4 \times 10^{-5} \mathrm{G}_{0}$ and, thus, remains reasonably close to the experimental value of $3 \times 10^{-4} \mathrm{G}_{0}$.

It has recently been shown that variations in the contact geometry between $\mathrm{C}_{60}$ and a $\mathrm{Cu}$ surface influenced the conductance with the largest conductance obtained for high coordination numbers. ${ }^{36}$ Since the structures we have considered are all maximally coordinated, as the molecule binds to a flat surface, one might expect to get lower conductances if the molecule instead binds to one or a few adatoms.

\section{CONCLUSION}

We have investigated the conductance of the dumbbell molecule BDC60 in a molecular junction with $\mathrm{Au}(111)$ electrodes. Adsorption on a $\mathrm{C}_{60}$ hexagon systematically gives a conductance very close to the experimental value $\sim 4$ $\times 10^{-4} \mathrm{G}_{0}$ while molecules adsorbed on a $6: 6$ bond have on average an order of magnitude lower conductance. Importantly, we find that within each class of adsorption type, the conductance is largely independent of geometry, which is varied through adsorption sites and rotation angles. Our calculations, thus, support the idea that $\mathrm{C}_{60}$ molecules will form a stable anchoring to the metal electrodes.

It was found that the conductance is mainly determined by the six unoccupied states LUMO to LUMO + 5 lying close in energy $0.2-0.5 \mathrm{eV}$ above the Fermi level. These six states are localized on either of the $\mathrm{C}_{60} \mathrm{~s}$ with a weak electronic coupling to the opposite electrode and a correspondingly low transmission value. Due to partial charge transfer, we argue that these states are pinned close to the Fermi level and, thus, reasonably well described with DFT methods. This is the origin of the very good agreement between our calculated conductance values and the experimental results.

\section{ACKNOWLEDGMENTS}

We acknowledge support from FTP Grant No. 274-080408 and The Danish Center for Scientific Computing. The 
center for Atomic-scale Materials Design (CAMD) is supported by the Lundbeck Foundation.

${ }^{1}$ A. Nitzan and M. A. Ratner, Science 300, 759 (2003).

${ }^{2}$ R. H. M. Smit, Y. Noat, C. Untiedt, N. D. Lang, M. C. van Hemert, and J. M. van Ruitenbeek, Nature (London) 419, 906 (2002).

${ }^{3}$ N. J. Tao, Nat. Nanotechnol. 1, 173 ( Dec. 2006).

${ }^{4}$ K. Moth-Poulsen and T. Bjornholm, Nat. Nanotechnol. 4, 551 (2009).

${ }^{5}$ A. H. Flood, J. F. Stoddart, D. W. Steuerman, and J. R. Heath, Science 306, 2055 (2004).

${ }^{6}$ H. Basch, R. Cohen, and M. A. Ratner, Nano Lett. 5, 1668 (2005).

${ }^{7}$ J. Ulrich, D. Esrail, W. Pontius, L. Venkataraman, D. Millar, and L. H. Doerrer, J. Phys. Chem. B 110, 2462 (2006).

${ }^{8}$ C. Li, I. Pobelov, A. Bagrets, A. Arnold, and F. Evers, J. Am. Chem. Soc. 130, 318 (2008).

${ }^{9}$ A. M. Moore, A. A. Dameron, B. A. Mantooth, R. K. Smith, D. J. Fuchs, J. W. Ciszek, F. Maya, Y. Yao, J. M. Tour, and P. S. Weiss, J. Am. Chem. Soc. 128, 1959 (2006).

${ }^{10}$ D. R. Jones and A. Troisi, J. Phys. Chem. C 111, 14567 (2007).

${ }^{11}$ I. S. Kristensen, D. Mowbray, K. S. Thygesen, and K. W. Jacobsen, J. Phys. Condens. Matter 20, 374101 (2008).

${ }^{12}$ M. Strange, O. Lopez-Acevedo, and H. HaÌkkinen, J. Phys. Chem. Lett. 1, 1528 (2010).

${ }^{13}$ J. A. Malen, P. Doak, K. Baheti, T. D. Tilley, A. Majumdar, and R. A. Segalman, Nano Lett. 9, 3406 (2009).

${ }^{14}$ C. A. Martin, D. Ding, J. K. Sørensen, T. Bjørnholm, J. M. van Ruitenbekk, and H. S. J. van der Zant, J. Am. Chem. Soc. 130, 13198 (2008).

${ }^{15}$ J. K. Sørensen, J. Fock, A. H. Pedersen, A. B. Petersen, K. Jennum, K. Bechgaard, K. Kilså, V. Geskin, J. Cornil, T. Bjørnholm, and M. B. Nielsen, J. Org. Chem. 76, 245 (2011).

${ }^{16}$ C. Rogero, J. I. Pascual, J. Gomez-Herrero, and A. M. Baro, J. Chem. Phys. 16, 832 (2002).

${ }^{17}$ J. Enkovaara, C. Rostgaard, J. J. Mortensen, J. Chen, M. Dulak, L. Ferrighi, J. Gavnholt, C. Glinsvad, V. Haikola, H. A. Hansen, H. H. Kristoffersen, M. Kuisma, A. H. Larsen, L. Lehtovaara, M. Ljungberg, O. Lopez-Acevedo, P. G. Moses, J. Ojanen, T. Olsen, V. Petzold, N. A. Romero, J. Stausholm-Moller, M. Strange, G. A. Tritsaris, M. Vanin,
M. Walter, B. Hammer, H. Hakkinen, G. K.H. Madsen, R. M. Nieminen, J. K. Norskov, M. Puska, T. T. Rantala, J. Schiotz, K. S. Thygesen, and K. W. Jacobsen, J. Phys Condens. Matter 22, 253202 (2010).

${ }^{18}$ G. Schull, N. Néel, M. Becker, J. Kröger, and R. Berndt, New J. Phys. 10, 065012 (2008).

${ }^{19}$ L. L. Wang and H. P. Cheng, Phys. Rev. B 69, 165417(12) (2004).

${ }^{20}$ C.-T. Tzeng, W.-S. Lo, J.-Y. Yuh, R.-Y. Chu, and K.-D. Tsuei, Phys. Rev. B 61, 2263 (2000).

${ }^{21}$ S. Datta, Electronic Transport in Mesoscopic Systems (Cambridge University Press, Cambridge, England, 1995).

${ }^{22}$ K. Thygesen and K. W. Jacobsen, Chem. Phys. 319, 111 (2005).

${ }^{23}$ A. H. Larsen, M. Vanin, J. J. Mortensen, K. S. Thygesen, and K. W. Jacobsen, Phys. Rev. B 80, 195112 (2009).

${ }^{24}$ J. P. Perdew, K. Burke, and M. Ernzerhof, Phys. Rev. Lett. 77, 3865 (1996).

${ }^{25}$ R. Haddon, L. Brus, and K. Raghavachari, Chem. Phys. Lett. 125, 459 (1986).

${ }^{26}$ C. Toher, A. Filippetti, S. Sanvito, and K. Burke, Phys. Rev. Lett. 95, 146402 (2005).

${ }^{27}$ N. Sai, M. Zwolak, G. Vignale, and M. Di Ventra, Phys. Rev. Lett. 94, 186810 (2005).

${ }^{28}$ A. Ferretti, A. Calzolari, R. Di Felice, F. Manghi, M. J. Caldas, M. B. Nardelli, and E. Molinari, Phys. Rev. Lett. 94, 116802 (2005).

${ }^{29}$ J. B. Neaton, M. S. Hybertsen, and S. G. Louie, Phys. Rev. Lett. 97, 216405 (2006).

${ }^{30}$ P. Darancet, A. Ferretti, D. Mayou, and V. Olevano, Phys. Rev. B 75, 075102 (2007).

${ }^{31}$ J. M. Garcia-Lastra, C. Rostgaard, A. Rubio, and K. S. Thygesen, Phys. Rev. B 80, 245427 (2009).

${ }^{32}$ S. Y. Quek, H. J. Choi, S. G. Louie, and J. B. Neaton, Nano Lett. 9, 3949 (2009).

${ }^{33}$ M. Strange, C. Rostgaard, H. Häkkinen, and K. S. Thygesen, Phys. Rev. B 83, 115108 (2011)

${ }^{34}$ D. J. Mowbray, G. Jones, and K. S. Thygesen, J. Chem. Phys. 128, 111103 (2008)

${ }^{35}$ E. Leary, M. T. González, C. van der Pol, M. R. Bryce, S. Filippone, N. Martín, G. Rubio-Bollinger, and N. Agraít, Nano Lett. 11, 2236 (2011).

${ }^{36}$ G. Schull, T. Frederiksen, A. Arnau, D. Sanchez-Portal, and R. Berndt, Nat. Nanotechnol. 6, 23 (2011). 